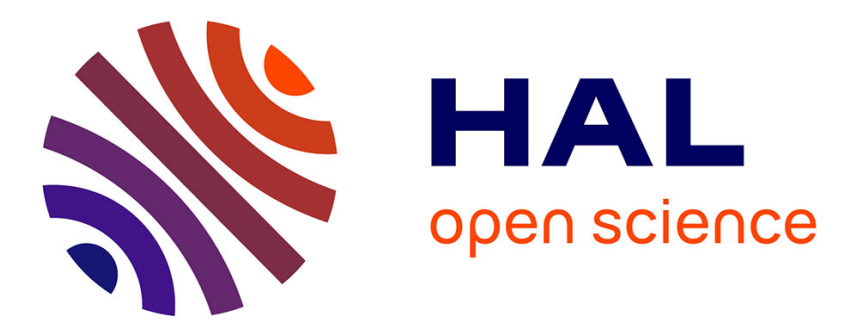

\title{
Le paradoxe de la mobilité: les évaluations subjectives des trajectoires postuniversitaires des diplômés chinois en France
}

Yong Li

\section{- To cite this version:}

Yong Li. Le paradoxe de la mobilité: les évaluations subjectives des trajectoires postuniversitaires des diplômés chinois en France. Migrations Société, 2020, 2020/2 (180), pp.97-112. 10.3917/migra.180.0097 . halshs-03274033

\section{HAL Id: halshs-03274033 \\ https://shs.hal.science/halshs-03274033}

Submitted on 5 Jul 2021

HAL is a multi-disciplinary open access archive for the deposit and dissemination of scientific research documents, whether they are published or not. The documents may come from teaching and research institutions in France or abroad, or from public or private research centers.
L'archive ouverte pluridisciplinaire HAL, est destinée au dépôt et à la diffusion de documents scientifiques de niveau recherche, publiés ou non, émanant des établissements d'enseignement et de recherche français ou étrangers, des laboratoires publics ou privés. 


\section{Le paradoxe de la mobilité}

\section{Les évaluations subjectives des trajectoires postuniversitaires des diplômés chinois en France}

LI Yong ${ }^{1}$

Triangle, ENS de Lyon

\section{Résumé}

En Chine, depuis une quinzaine d'années, dans un contexte d'inflation des diplômes et de crise de l'emploi, la signification des études à l'étranger a profondément changé pour les jeunes générations. Jadis garantie des positions d'élite en Chine, un diplôme étranger perd aujourd'hui une grande partie de son exceptionnalité statutaire. C'est ainsi que beaucoup d'étudiants chinois en mobilité s'efforcent d'acquérir une expérience de travail dans leur pays de formation avant de retourner dans leur pays d'origine. Ils espèrent améliorer leur employabilité par l'épreuve du marché du travail étranger. Notre contribution se focalise sur les trajectoires des diplômés chinois qui exercent un emploi en France après leurs formations françaises. À partir d'une enquête par récits de vie, nous mettrons en relief le sentiment d'immobilité des diplômés enquêtés et leur évaluation ambivalente quant au succès de leur migration. En effet, l'allongement de la scolarité dans un pays étranger impose aux jeunes diplômés un coût non négligeable en termes de temps et d'argent. À l'aune des normes d'âge chinoises, leur entrée dans la vie active est retardée, ce qui compliquerait leur installation dans la vie adulte, notamment pour les femmes. Plus profondément, dans un contexte de montée en puissance de la Chine et de stagnation économique en Occident, l'avancement d'une carrière professionnelle paraît toujours plus lent et plus difficile à l'étranger que dans son pays d'origine. Beaucoup de diplômés se sentent en décalage avec leurs pairs restés en Chine. Pour beaucoup d'enquêtés, l'entrée dans la vie adulte en France, ainsi que le sentiment de double décalage (par rapport à son idéal de réussite et par rapport à autrui) qu'ils éprouvent durant leur migration, expliquent en grande partie la révision de leurs projets de migration. Ceux-ci s’articulent de plus en plus autour d'un ancrage durable en France au lieu d'un retour définitif en Chine. En analysant cette immobilité paradoxale dans la mobilité pour études, nous souhaitons souligner l'importance de placer les trajectoires des étudiants dans le processus biographique plus général et d'étudier les effets des changements sociaux sur les parcours individuels.

Mots-clés : diplômés chinois, trajectoires postuniversitaires, sentiment d'immobilité, entrée dans la vie adulte, changements sociaux

${ }^{1}$ Docteur en sociologie, postdoctorant au laboratoire Triangle (Action, Discours, Pensée politique \& économique), École normale supérieure de Lyon. 


\begin{abstract}
In China, over the past fifteen years, in the context of educational inflation and job crisis, the meaning of studying abroad has profoundly changed for the younger generations. Formerly a guarantee of elite positions in China, a foreign diploma today loses a large part of its statutory exceptionality. Thus, many Chinese students in France try to gain work experience in their host country before returning to their home country. They hope to improve their employability through the ordeal of the French labor market. This paper focuses on the trajectories of Chinese graduates who work in France after their French training. Based on a life story survey, I will highlight the feeling of immobility of the graduates surveyed and their ambivalent assessment of the success of their migration. Indeed, the extension of college studies in a foreign country imposes on students a significant cost in terms of time and money. According to Chinese norms related to age, their entry into working life is delayed, which would complicate their settlement in family life, especially for women. More profoundly, in a context of the rise of China and economic stagnation in the West, the advancement of a professional career always seems slower and more difficult abroad than in one's country of origin. Many graduates feel out of step with their peers who have stayed in China. For many graduates, their entry into adult life in France, as well as the double gap (in relation to one's ideal of success and in relation to others) that they experience during their migration largely explain their modification of their migration plans. These revolve more and more around a lasting anchorage in France instead of a definitive return to China. By analyzing this paradoxical immobility in mobility for studies, I wish to emphasize the importance of placing the trajectories of students in the more general biographical process as well as that of studying the effects of social changes on individual life-courses.
\end{abstract}

Keywords: Chinese graduates, post-university trajectories, feeling of immobility, transitions to adulthood, social changes

\title{
Introduction: Migration et évaluation subjective de la mobilité
}

En Chine, depuis une quinzaine d'années, le contexte historique pour penser la mobilité des étudiants chinois a radicalement changé. $\mathrm{Si}$, dans les années qui ont suivi la réforme et l'ouverture de la Chine (depuis 1978), les candidats aux études à l'étranger étaient majoritairement boursiers du gouvernement chinois et un retour avec un simple diplôme étranger était suffisant pour assurer au diplômé rapatrié un bon travail ; aujourd’hui, avec la massification des départs autofinancés (depuis la fin des années 1990), l'accélération des retours et le chômage des diplômés à la hausse, le marché du travail chinois a adopté une attitude beaucoup plus discriminante envers le recrutement des diplômés internationaux ${ }^{2}$. En France, huitième pays d'accueil d'étudiants chinois en mobilité, les jeunes chinois sont nombreux à recourir à une stratégie du «retour différé» pour résister à un déclassement anticipé sur le

\footnotetext{
${ }^{2}$ HAO, Jie ; WELCH, Anthony, “A tale of sea turtles: Job-seeking experiences of haigui (high-skilled returnees) in China”, Higher Education Policy, vol. 25, n² 2,2012, pp. 243-260.
} 
marché du travail chinois. À l'issue de leurs études, certains diplômés cherchent à acquérir une première expérience professionnelle en France afin d'obtenir une meilleure représentation de leur employabilité pour leurs futurs employeurs. Or, durant leurs années de travail en France, beaucoup d'anciens étudiants chinois ont l'impression de s'éloigner des normes de la réussite du pays d'origine, de prendre de plus en plus de retard par rapport à ceux qui sont restés sur place. Ils ne peuvent éviter d'éprouver un sentiment d'immobilité dans le processus de mobilité internationale. Comment comprendre ce paradoxe ?

En partie pour surmonter les difficultés ${ }^{3}$ que pose une approche conventionnelle de la mobilité sociale centrée sur les trajectoires objectives des individus ${ }^{4}$, depuis une vingtaine d'années, de plus en plus de chercheurs s'intéressent à la dimension subjective de la mobilité sociale ${ }^{5}$. Mais l'étude sur la dimension subjective de la mobilité, entendue comme la manière dont les individus vivent et expliquent leurs trajectoires sociales ${ }^{6}$, pose aussi une série de questions : quel est le groupe de référence choisi par les individus dans leur évaluation des trajectoires ${ }^{7}$ ? Quels sont précisément ces facteurs subjectifs qui jouent dans les jugements des migrants quant au succès et l'échec de leur migration ? Comment le contexte de migration influe-t-il sur les trajectoires des migrants et leurs auto-évaluations ?

Les théories classiques de comparaison sociale considèrent que des personnes similaires à un individu sont particulièrement utiles à celui-ci pour générer des évaluations précises de ses capacités et opinions ${ }^{8}$. En général, la recherche existante sur les migrations de travail montre comment les migrants se comparent souvent à leurs pairs dans les pays d'origine pour mesurer la réussite ou l'échec de leur migration ${ }^{9}$. Dans les migrations qui visent à une ascension sociale, les motivations de migrer sont souvent nées de la comparaison des migrants avec leurs semblables, par exemple, les voisins de village pour les migrants mexicains partant aux ÉtatsUnis étudiés par Stark Oded et Taylor J. Edward ${ }^{10}$. Une fois arrivés à leur destination, de nombreux migrants continuent à se référer à leurs compatriotes restés au pays pour donner sens

\footnotetext{
3 Voir Beaud, Stéphane ; Pasquali, Paul, “Ascenseur ou descenseur social ? Apports et limites des enquêtes de mobilité sociale”, Cahier Français, La Documentation française, n 383, 2014, pp.19-25 (voir p.24).

4 Bertaux, Daniel; Thompson, Paul (sous la direction de), Pathways to social class: A qualitative approach to social mobility, Oxford: Oxford University Press, 1997, 344 p.

${ }^{5}$ Attias-Donfut, Claudine ; François-Charles, Wolff, “ La dimension subjective de la mobilité sociale”. Population, vol.56, n 6, 2001, pp. 919-958.

6 Duru-Bellat, Marie ; Kieffer, Annick, “Les deux faces - objective/subjective - de la mobilité sociale”, Sociologie du Travail, vol.48, $\mathrm{n}^{\circ}$ 4, 2006, pp. 455-473.

7 Lévy, Florence; Li, Yong (2019). Avancer ou régresser? Comparer les évaluations subjectives des trajectoires professionnelles de migrant.e.s chinois. e. s en France depuis les années 2000, communication présentée au 9e Congrès AFS 2009, Aix-en-Provence.

${ }^{8}$ Festinger, Leon, “A Theory of Social Comparison Processes”. Human Relations, vol.7, n 2, 1954, pp.117-140; Evans, M. D. R.; Kelley, Jonathan. “Subjective sociallocation: data from 21 nations”. International Journal of Public Opinion Research, vol.16, $\mathrm{n}^{\circ}$ 1, 2004, pp.3-39; Merton, Robert King, Social theory and social structure (enlarged ed.). New York: The Free Press, 1968, 713p.
}

9 Nowicka, Magdalena, "Successful Earners and Failing Others: Transnational Orientation as Biographical Resource in the Context of Labor Migration”. International Migration, vol.52, n 1, 2014, pp.74-86.

10 Stark, Oded; Taylor, J. Edward, “Relative Deprivation and International Migration”, Demography, vol.26, $n^{\circ} 1$, 1986, pp.1-14. 
à leur migration ${ }^{11}$. Ces perspectives justifient qu'on accorde une attention particulière au rôle du groupe des pairs non-migrant dans l'évaluation subjective des migrants.

Durant leur séjour à l'étranger, les migrants étudiants doivent répondre non seulement aux attentes sociales de réussir leurs études et l'insertion professionnelle subséquente, mais aussi aux normes sociales de «devenir adulte » spécifiques à un contexte sociohistorique. La recherche sur la question de la jeunesse et du passage à la vie adulte est foisonnante, notamment en ce qui concerne les jeunes Européens en mobilité dans le contexte de l'intégration européenne et de la libre circulation ${ }^{12}$. D’un côté, la mobilité affecte la «temporalité », «l'espace» et le «rythme des biographies » des jeunes qui s'y sont engagés. Elle peut redoubler la précarité des jeunes et compliquer leurs itinéraires de transition vers l'âge adulte ${ }^{13}$. De l'autre, les expériences de transition juvéniles tendent à façonner les expériences de mobilité des jeunes ${ }^{14}$. C'est ainsi que beaucoup de jeunes migrants restent attachés à une conception normative de l'âge adulte- - en ce qui concerne le timing de la transition, mais aussi son ordre séquentiel (accès au travail, autonomie financière et indépendance résidentielle, etc.) —-même si en réalité, ils ont peu de chance de s'y conformer dans un contexte de crise économique et sociale ${ }^{15}$. Nous pouvons regretter que cette dimension de la transition juvénile soit rarement abordée dans les travaux existants sur la mobilité des étudiants chinois, à quelques exceptions près. Issus d'un pays où les normales sociales régissant le passage à l'âge adulte sont assez contraignantes, confrontés à la précarité statutaire et aux incertitudes en situation de migration, les jeunes migrants chinois ne se retrouvent-ils pas dans une situation particulièrement délicate pour gérer leurs multiples transitions à l'issue de leurs formations en France?

Toutefois, la difficulté des jeunes en mobilité est aussi de se construire dans un contexte sociohistorique qui ne cesse lui-même de changer. Dans une perspective macrosociologique, les transformations sociétales modifient le système de stratification sociale et par conséquent, constituent des facteurs déterminants des trajectoires ascendantes et descendantes des migrants. Dominique Vidal montre comment le rattrapage économique du Portugal depuis les années 1980 influe sur le positionnement social des migrants portugais anciennement installés en France par rapport à leurs milieux d'origine ${ }^{16}$. Shen et Xiang montrent comment dans un contexte de la solidification des frontières de classes en Chine, l'éducation internationale favorise aujourd'hui la reproduction des positions de classe des jeunes chinois en mobilité,

11 Cette perspective n'exclut pas le fait que le choix du groupe de référence par les migrants tend à varier selon leur durée d’installation et leur position générationnelle. Voir Lönnqvist, Jan-Erik, Jasinskaja-Lahti, Inga, \& Verkasalo, Markku,“Personal values before and after migration: A longitudinal case study on value change in Ingrian-Finnish migrants”, Social Psychological and Personality Science, vol.2, $\mathrm{n}^{\circ}$ 6, 2011, pp.584-591.

12 Flipo, Aurore, “Mobilité et passage à l’âge adulte. Itinéraires de migrants intra-européens”,Agora débats/jeunesses, vol.65, n³, 2013, pp.23-35.

13 Bidart, Claire (sous la direction de), Devenir adulte aujourd'hui : perspectives internationales. Paris: L’Harmattan/INJEP, 2006, 232p.

14 Collins, Francis L.;\& Shubin, Sergei, “The temporal complexity of international student mobilities”, in: Mavroudi, Elizabeth; Page, Ben; Christou, Anastasia (Ed.), Timespace and international migration, Cheltenham: Edward Elgar Publishing, 2017, pp.17-32.

15 C'est le cas des jeunes migrants italiens en Grande-Bretagne étudiés par Varriale, Simone. "Unequal Youth Migrations: Exploring the Synchrony between Social Ageing and Social Mobility among Post-Crisis European Migrants”, Sociology, vol.53, n6, 2019, pp. 1160-1176.

16 Vidal, Dominique, Pourquoi et comment étudier les migrations à l'échelle de l'individu ? Le cas de l'immigration portugaise en France, communication présentée au 7e Congrès de l’AFS, Amiens, 2017. 
plutôt que leur ascension sociale ${ }^{17}$. Ce qui prime ainsi, à notre sens, ce sont moins les transformations sociales per se que la vitesse ou le rythme de ces transformations. Car les transformations sociétales rapides sont susceptibles de bousculer la hiérarchie sociale existante, à mettre à mal les anciennes modalités de la réussite sociale des migrants et leurs anciennes formes d'identification. Se posent ainsi les questions de l'interaction entre les rythmes de la vie personnelle et les rythmes de société ${ }^{18}$. De ce point de vue, les étudiants migrants chinois en France peuvent constituer un cas d'étude heuristique. Il nous permettrait d'examiner les manières dont les transformations radicales de la Chine, survenues au cours du séjour des migrants étudiants chinois en France influent sur leur mobilité subjective et leurs identités personnelles.

Ainsi, dans cet article, nous nous efforçons de construire une approche de la mobilité subjective ${ }^{19}$ prenant en compte le groupe de référence et sensible à la temporalité de migration ${ }^{20}$, afin de comprendre les évaluations subjectives des migrants étudiants chinois à propos de leurs trajectoires postuniversitaires. Notre article vise à comprendre ce paradoxe de "l'immobilité dans la mobilité » en élucidant ses mécanismes de production et ses effets sur les projets migratoires individuels. Les matériaux empiriques de cet article s’appuient sur les récits de vie recueillis dans le cadre d'une thèse de doctorat portant sur l'insertion professionnelle des diplômés chinois en France ${ }^{21}$. Entre 2007 et 2013, nous avons interrogé au total 45 diplômés chinois (23 hommes et 22 femmes) qui travaillaient ou qui cherchaient du travail en Normandie et en région parisienne : 24 salariés et stagiaires, 5 chercheurs scientifiques, 9 entrepreneurs/commerçants et 7 autres diplômés ayant d'autres statuts (chercheurs d'emploi, inactif, professions libérales, etc.). Nos enquêtés sont âgés de 25 à 36 ans et sont arrivés en France au début ou au milieu des années 2000 avec leur propre financement. La durée moyenne de leur séjour en France est de 7 ans. Les salariés possèdent tous un diplôme de niveau Bac +5 , dans une grande école ou dans une filière universitaire valorisante. Ils sont ingénieurs en service numérique, cadres d'audit, comptables, paysagistes et occupent, pour la plupart d'entre eux, un CDI dans une entreprise française. Les chercheurs sont tous nouveaux docteurs ou postdoctorants en sciences de la nature et œuvrant dans les laboratoires français.

Les diplômés chinois enquêtés ont, pour bon nombre d’eux, effectué une partie de leurs études supérieures en Chine, mais restaient insatisfaits de la qualité de leur formation ou du prestige

\footnotetext{
17 Xiang, Biao ; Shen, Wei, “International student migration and social stratification in China”, International Journal of Educational Development, vol.29, $\mathrm{n}^{\circ}$ 5, 2009, pp.513-522. Les recherches comparatives montrent que cette solidification des frontières de classe est toute relative et les mobilités sociales (hiérarchiques et sectorielles) sont toujours plus fréquentes en Chine que dans les pays capitalistes avancés comme la France. Zhou, Xiang; Xie, $\mathrm{Yu}$, "Market Transition, Industrialization, and Social Mobility Trends in Postrevolution China”, American Journal of Sociology, vol.124, $\mathrm{n}^{\circ}$ 6, 2019, pp.1810-1847.

${ }_{18}$ Lefebvre, Henri. Éléments de Rythmanalyse : Introduction À la Connaissance des Rythmes. Paris: Eterotopia, 1992, 116 p.

19 Van den Berg, Marguerite, “Subjective social mobility: Definitions and expectations of 'moving up' of poor Moroccan women in the Netherlands”. International Sociology, vol.26, n 4, 2011, pp.503-523.

20 Van Geel, Joan; Mazzucato, Valentina, “Conceptualising youth mobility trajectories: thinking beyond conventional categories”. Journal of Ethnic and Migration Studies, vol.44, n 13, 2017, pp.1-19.

$21 \mathrm{Li}$, Yong, Condamnés à réussir. Insertion professionnelle des diplômés chinois en France, nouvelles dynamiques migratoires et identitaires (thèse de doctorat), Université de Rouen,2016, 695 p. Nous avons recueilli 45 récits de vie auprès des diplômés chinois qui sont restés en France après leurs études, essentiellement pour le motif professionnel.
} 
de leur université et souhaitaient valoriser leurs cursus par des études à l'étranger. Issus majoritairement des classes moyennes éduquées (cadres, techniciens, personnels administratifs, chefs d'entreprise...), nos enquêtés incarnent cette jeunesse chinoise qui a intériorisé l'idéologie du culte de l'excellence via l'investissement dans le capital humain ${ }^{22}$. À travers la migration, nos enquêtés veulent se stimuler, se mettre à l'épreuve, afin de devenir plus performants, et espèrent être récompensés à leur retour en Chine par l'accès à des positions professionnelles prometteuses. Ainsi, la plupart d'entre eux n'avaient pas l'intention de s'installer en France et considéraient leurs années de travail en France comme un tremplin vers le monde du travail chinois.

\section{La difficile « entrée dans la vie adulte » en situation d'immigration}

En Chine comme en Europe, on assiste à une « dé-temporalisation » du parcours de vie individuel $^{23}$. Les âges du premier mariage reculent et la transition vers l'âge adulte ne semble plus être encadrée par des repères temporels précis. Or, la conception normative d'une biographie, ce que certains auteurs appellent une « biographie normale $»^{24}$, continue à peser un grand poids sur les jeunes, même s'ils doivent faire face à un contexte social très différent de celui de la génération de leurs parents. Ainsi, les jeunes chinois d'aujourd'hui sont toujours confrontés aux injonctions parentales de réussir dans leur passage à l'âge adulte à temps et en bon ordre : études, insertion professionnelle, mariage, parentalité. Toutefois, les normes d’âge sont différentes pour les deux sexes : si les hommes chinois peuvent s’autoriser un certain retard pour entrer dans la relation matrimoniale (jusqu’à 34 ans), au profit de leurs études ou de leur carrière professionnelle, ce n'est pas le cas pour les femmes chinoises, qui dépassant un certain « âge limite» (en général 28 ans), sont socialement stigmatisées ${ }^{25}$.

Cependant, de nombreux jeunes chinois en mobilité pour études se retrouvent de facto dans une situation déviante au regard des normes d’âge dominantes en Chine. En effet, l’allongement de la scolarité dans un pays étranger impose aux jeunes diplômés un coût non négligeable en termes de temps et d'argent ${ }^{26}$. Durant leurs études, les jeunes Chinois que nous avons rencontrés ont l'impression d'être figés dans leurs situations de départ (étudiant, célibataire, inactif, etc.), pendant que leurs pairs non migrants accèdent à l'autonomie économique, aident financièrement leurs parents, progressent dans leur vie professionnelle et familiale. Dès lors, les migrants se voient attribuer un statut de «cadette sociale» et se sentent déclassés face à leurs pairs. Ce sentiment de stagnation concerne presque tous les enquêtés, mais affecte en

\footnotetext{
${ }^{22}$ Roulleau-Berger, Laurence; Yan, Jun, Travail et migration. Jeunesses chinoises à Shanghai et Paris. La Tour d’Aigues : Editions de l'aube, 2017, 223p.

23 Rosa, Hartmut, "Social Acceleration: Ethical and Political Consequences of a Desynchronized High-Speed Society”. Constellations, vol.10, n 1, 2003, pp.3-33.

${ }^{24}$ Kohli, Martin, “The Institutionalization of the Life Course: Looking Back to Look Ahead”, Research in Human Development, vol. 4, n³-4, 2007, pp.253-271.

25 Wang, Simeng, "Des «soucis matrimoniaux» dans le contexte transnational : le cas des migrants et des migrantes chinois qualifiés à Paris”, op. cit., voir p. 160.

${ }^{26}$ Boudon, Raymond, L'inégalité des chances : la mobilité sociale dans les sociétés industrielles, Paris : Armand Colin, 1973, 209 p.
} 
particulier les jeunes engagés dans des cursus longs (master, doctorat) ou ayant connu des piétinements dans leurs études. À ce sujet, Yang, 30 ans, docteur en génie des matériaux, témoigne :

«Au début de mes études, je n’avais jamais pensé que j’aurais des problèmes financiers, car j'avais une bourse ${ }^{27}$. Mais après j'ai trouvé que la bourse était très insuffisante. Cet argent me permet seulement de vivre tout seul à l'étranger. Quand on devient plus âgé, les familles auront besoin de notre aide. À ce momentlà, vous découvrez que vous ne pouvez que gagner difficilement votre vie et que vous n'avez pas la capacité d'aider les autres. Mais vos parents et votre famille comptent sur vos aides, car ils ont vu que vous avez fait beaucoup d'études. Ils ont vu aussi que vos camarades ont déjà leurs enfants, qu'ils ont pris en charge leurs parents... Mais vous restez toujours ici tout seul. Ça, ce n'est pas bien vu. Et en plus, c'est difficile d'obtenir le diplôme. J'ai donc la pression sur tous les plans ». (9 mai 2009)

À cet allongement de la scolarité s’ajoutent les difficultés rencontrées par nos enquêtés pour accéder à l'emploi à l'issue de leurs études, et corrélativement, à un statut administratif stable (un titre de séjour de travail au lieu d'un titre de séjour étudiant). Nous savons comment les diplômés étrangers, lorsqu'ils deviennent candidats à l’immigration, sont fréquemment exposés aux verrouillages institutionnels et aux aléas sur le marché du travail dans le pays d'accueil ${ }^{28}$. La procédure de changement de statut tend à engendrer des zones de déstabilisation, d'incertitude, de quête, de flou, sorte de no man's land social que Arnold Van Gennep a traduit par le terme de « zone de liminarité ${ }^{29}$. Cette instabilité professionnelle empêche les migrants étudiants chinois de se projeter dans un avenir clair. De ce fait, l'entrée du migrant dans la vie adulte se trouve retardée. Les relations amoureuses sont souvent déstabilisées ou reléguées au second plan au profit de la carrière professionnelle ; la mise en ménage et la conception d'un enfant sont ajournées. Dès lors, le migrant a l'impression d'accumuler du retard dans les différents domaines de la vie par rapport à ses pairs restés au pays.

Au moment de l'entretien, Ting (30 ans, DESS administration des entreprises) voulait se marier rapidement avec son compagnon Zheng (32 ans, informaticien dans une grande entreprise de service informatique) et faire des enfants. Ting envisageait aussi de faire venir ses parents en France à l'occasion de son mariage. Mais sa situation administrative l'empêchait de s’y préparer. Titulaire d'une carte de séjour « travailleur temporaire », au chômage depuis 7 mois, Ting était obligée de trouver un emploi afin de pouvoir renouveler son titre. Stressée par la recherche d’emploi, Ting a décidé de reporter son mariage, et toute une série de projets, entre autres la parentalité et la venue de ses parents en France. On voit comment les évènements critiques qui touchent la migrante dans sa vie professionnelle influent sur sa vie conjugale, et comment les trajectoires interfèrent au sein d'un couple. Zheng disait :

\footnotetext{
27 Yang bénéficiait d'une allocation de recherche octroyée par la région Normandie.

${ }^{28}$ Li, Yong. "La confrontation des diplômés chinois au marché du travail français. Une insertion incertaine ?" Connaissance de l'emploi, $\mathrm{n}^{\circ} 145$, janvier 2019.

${ }^{29}$ Van Gennep, Arnod, Les rites de passage : étude systématique. Paris : Picard, 1981 (Réimpr. De l’édition 1909), 320 p. Cité par : Gohard-Radenkovic, Aline, “Contre-point. Quand la toute-mobilité peut devenir l'immobilisation des acteurs de la mobilité... et quand sociétés d'accueil et de départ produisent du « brain waste » ”, Journal of international Mobility, vol. 5, n¹, 2017, pp. 157-176.
} 
« Ici, en France, on rencontre toutes sortes de problèmes : le séjour, le travail, la langue, des questions sociales, l'intégration. Il faut réussir dans tous les domaines pour penser sérieusement au mariage » (6 décembre 2008).

Mais les difficultés professionnelles et administratives ne sont pas les seuls facteurs explicatifs du célibat et du mariage tardif de nos enquêtés. Les salariés en CDI qui jouissent d'une situation administrative plutôt stable rencontrent aussi des soucis matrimoniaux, liés plus particulièrement à la configuration du marché matrimonial en France. Les migrants chinois qualifiés cherchent avant tout leurs futurs conjoints parmi leurs compatriotes, à travers leurs réseaux de sociabilité, ce qui limite objectivement leurs opportunités de rencontre. De plus, la relation de genre tend à se modifier en situation de migration: les migrantes enquêtées possèdent aussi une ambition professionnelle et ne souhaitent pas se confiner à leur rôle d'épouse et de mère de famille ${ }^{30}$. Pour les migrants des deux sexes, le fait de vivre à l'étranger rend plus difficile la réalisation d'un mariage idéal selon les normes chinoises, autrement dit une répartition des rôles où le mari est pourvoyeur économique de la famille, tandis que l'épouse prend en charge les tâches domestiques et l'éducation de l'enfant. Paradoxalement, les migrants chinois les plus qualifiés sont aussi ceux qui sont les plus tourmentés par des «soucis matrimoniaux $»^{31}$.

Bo, 30 ans, est employé dans une grande banque. Célibataire, il prend très au sérieux le mariage :

« Nous avons dépassé la période de 20-25 ans. Avant, quand on rencontrait quelqu'un, on sortait ensemble et on se séparait. On faisait des rencontres autant de fois qu'on voulait. On n'est plus là. On cherche vraiment la stabilité, et on a de choix très limité : soit parmi ses amies, soit parmi les amies de ses amies. C’est peut-être pourquoi il y a autant de célibataires dans ce métier (celui de la banque). Mon ami, qui travaille à son compte, est plus âgé que nous — il a 35 ans — mais il est toujours célibataire. »

\section{Vivre sa vie au ralenti à l'étranger}

Selon John Urry ${ }^{32}$, à l'échelle mondiale, il existe des voies rapides qui accélèrent le tempo de la vie individuelle, tout comme des voies lentes qui «ralentissent» le rythme de développement de la vie individuelle. Lorsque les étudiants chinois sont en France, ils ont l'impression d'être captés dans une voie lente de la vie sociale, tandis que leurs pairs en Chine avancent dans une voie rapide de la vie sociale.

\footnotetext{
30 C'est pour cette raison que Ting ne veut pas solliciter une carte «vie privée et familiale » qui l'autorise à travailler, mais qui la rend dépendante administrativement de son mari. Elle préfère obtenir un titre de séjour de travail et affirmer son autonomie.

31 Simeng Wang, "Des «soucis matrimoniaux» dans le contexte transnational : le cas des migrants et des migrantes chinois qualifiés à Paris”, op. cit. montre que les inquiétudes matrimoniales constituent une des causes principales des souffrances psychiques chez les migrants qualifiés chinois à Paris.

${ }^{32}$ Urry, John, “Les systèmes de la mobilité”, Cahiers internationaux de sociologie, vol.118, n 1, 2005, pp.23-35.
} 
La différence entre la Chine et la France en termes de rythme de changements sociaux est suffisamment grande pour avoir un impact sur les trajectoires sociales des étudiants chinois en mobilité. Pendant que nos enquêtés étudiaient et travaillaient en France - ce qui correspond grosso modo à la première décennie des années 2000 - avec un taux de croissance annuel de $11,4 \%$, le PIB chinois a plus que doublé, tandis que le PIB français n’a augmenté que très légèrement ${ }^{33}$. La montée en puissance des classes moyennes chinoises, et le déclin relatif des classes moyennes des pays développés, si bien illustrés dans la fameuse «courbe de l'éléphant ${ }^{34}$, trouvent des échos dans les vécus de nos enquêtés. Les camarades de nos enquêtés qui ont commencé à travailler immédiatement après leurs études universitaires chinoises, au lieu de partir à l'étranger, sont devenus grands gagnants dans cette conjoncture particulièrement favorable. Certains ont vu leurs revenus plus que doubler en l'espace de quelques années; d'autres sont rapidement montés en grade et ont accédé aux postes à responsabilité ; d'autres encore ont acheté leurs logements dans de grandes villes chinoises et se sont enrichis après la flambée du prix immobilier. Dans ce contexte, nos enquêtés qui peinent à évoluer dans les entreprises françaises ${ }^{35}$ sont largement devancés par leurs amis en Chine tant du point de vue des revenus que des responsabilités professionnelles. Dong, 26 ans, diplômée de Télécom ParisTech, ingénieure réseaux dans une grande entreprise française, se dit étonnée par l'avancée professionnelle de ses camarades de l'université qui sont restés en Chine. La comparaison avec ses amis en Chine l'amène à s'interroger sur le bien-fondé de son choix de venir étudier en France :

« Mais parfois je les trouve très courageux et forts. Comment peuvent-ils gagner autant dans le contexte chinois ? C'est-à-dire plusieurs centaines de milliers yuans par an ${ }^{36}[\ldots]$ Je pense que si je n'étais pas venue en France, si j’avais commencé à travailler immédiatement après mes études, j’aurais pu avoir aussi un très bon développement. Mais je n’ai pas trouvé de bonnes opportunités à ce moment-là, j’ai donc choisi d'aller étudier à l'étranger. Ça dépend de la situation de chacun. J'ai des amis qui m’ont dit, “pourquoi tu es allée à l'étranger? Sans sortir du pays, tu peux aussi avoir un bon avenir. Regarde, moi, je ne gagnais seulement 2000 yuans au début, maintenant, j'ai un salaire confortable”». (27 mai 2007)

La transformation rapide de la société chinoise s’accompagne des changements radicaux sur le marché du travail et dans les différents domaines de la vie sociale. Ainsi, les étudiants qui misaient sur la rareté des diplômes étrangers pour accéder à des positions d'élite en Chine découvrent quelques années après que ce diplôme n’est plus un sésame efficace. De même, les compétences rares et recherchées sur le marché du travail peuvent se banaliser et perdre de leurs valeurs en l'espace de quelques années. Ces changements mettent à mal les stratégies

\footnotetext{
33 Selon les données de l'INSEE, le taux de croissance annuel en France est de 1,64 \% durant les années 20002010, soit une augmentation de $17,5 \%$ en une décennie.

34 Dessinée par l'économiste Branko Milanovic, cette courbe montre la redistribution de revenus à l'échelle mondiale : Milanovic, Branko, Global Inequality: A New Approach for the Age of Globalization, Cambridge: The Belknap press of Harvard University press, 2016, 320 p.

${ }^{35}$ Cette évolution se caractérise par une faible augmentation de salaire (le taux d'augmentation annuel est de $2 \%$ $3 \%$ pour la plupart de salariés interrogés) et une faible chance d'accéder aux postes d'encadrement.

36 Au moment de l'enquête, un euro équivalait 8 yuans. À titre de comparaison, Dong percevait un salaire net annuel de 24000 euros, soit 192000 yuans.
} 
d'accumulation des expériences professionnelles des diplômés chinois et les obligent à modifier constamment leurs projets de migration.

C’est l'expérience que fait Zhou, venu en France en 2003 pour étudier le design de paysage. Il a terminé son cursus pendant que son épouse était encore en formation doctorale. Il est entré dans une entreprise normande d'urbanisme tout en souhaitant retourner en Chine après quelques années d'expérience professionnelle. En 2013, alors qu'il se sent prêt à rentrer en Chine, il constate que les conditions socioéconomiques ont radicalement évolué dans l’intervalle. En 1998, lorsqu'il était étudiant en Chine, le design de paysage par ordinateur était une compétence rare. Une mission était payée 5000 yuans (équivalent à 625 euros). Au même moment, le prix de l'immobilier était bas, puisqu'il fallait compter 1000 yuans (équivalent à 125 euros) par mètre carré au centre de Hangzhou, sa ville natale. En 2013, il constate qu' «il n’y a pas de logement à moins de 20000 yuans par mètre carré au centre-ville». De plus chaque année, beaucoup de nouveaux diplômés entrent dans le métier et la concurrence s’intensifie. Un rendu vaut 500 à 1000 yuans. Zhou considère avoir raté le bon moment pour retourner en Chine. Il a perdu tout espoir de rivaliser avec ses anciens camarades d'université qui sont restés au pays et sont devenus directeurs d'instituts d'architecture. Son projet est donc de travailler en France en espérant un jour créer son propre cabinet d'architecture.

Le changement est tout aussi drastique sur le marché scientifique du travail en Chine. Au début du nouveau millénaire, pour stimuler davantage sa capacité d'innovation, le gouvernement chinois a soulevé la barre des dispositifs de recrutement des talents d'outre-mer de haut niveau, comme l'illustrent les deux programmes récents : Le programme d’introduction des talents axés sur des disciplines aux universités (Projet 111) lancé en 2006 ; le programme Mille Talents initié en $2008^{37}$. Un système de classification des talents d'outre-mer a été institutionnalisé ${ }^{38}$. Les jeunes chercheurs formés en France, qui pouvaient autrefois compter sur les programmes d'introduction des talents d'outre-mer plus ouverts pour retourner en Chine, se trouvent aujourd'hui confrontés à une logique de sélection très élitiste. Devant cette exigence implacable d'excellence, aucun des jeunes chercheurs que nous avons interrogés ne se considère comme éligible aux programmes officiels de «talents de haut niveau ». Certains étaient obligés de réviser leurs attentes vers le bas et chercher un poste dans des établissements chinois de deuxième ou de troisième rang. D’autres ont reporté leur retour en Chine et continué à travailler dans un laboratoire étranger en espérant accumuler plus d'expériences internationales.

\section{Le double décalage et la modification du projet de migration}

Nouveauté historique dans les mouvements migratoires entre la Chine et le monde développé, aujourd’hui, de nombreux migrants chinois en France éprouvent un sentiment de

\footnotetext{
37 Welch, Anthony; Cai, Hao, “Enter the Dragon: The internationalization of China’s higher education system”, in Janette Ryan (Ed.), China’s higher Education Reform and Internationalization. Abingdon, UK: Routledge, 2011, pp. 9-33.

${ }^{38}$ La définition d'un «talent de haut niveau » tient compte non seulement de sa compétence professionnelle per se, mais aussi du statut international de son pays de formation ou de travail, du prestige de son institution d'affiliation, de la réputation de son cursus universitaire, etc. Dans le cas des chercheurs, il y a une préférence pour les expériences étatsuniennes, et pour les publications à haut facteur-impact dans les revues anglophones.
} 
privation relative, une amertume, lorsqu'ils comparent leurs situations avec celles de leurs pairs qui ont le mieux réussi en Chine.

En admettant qu'il ait la «honte» de se comparer avec ses amis en France, Zheng considère que son expérience est commune à toute une génération de jeunes chinois en mobilité :

«Pour mes amis qui sont dans le commerce, ils sont déjà directeurs de leurs entreprises ; pour ceux qui sont dans le monde politique, ils sont déjà chefs de section ou chefs de département. Ils peuvent monter en grade étape par étape. Ils ont déjà leur famille, leurs enfants. Tout le monde a son logement ; la plupart ont leur voiture. Ils sont en train d'entrer dans la classe moyenne. [...] Nous avons passé 6 ans en France pour étudier à l'université, pour revenir dans l'école d'ingénieurs ${ }^{39}$, pour travailler. En fait, nous n'avons aucun avantage par rapport à nos camarades en Chine $[\ldots]$

Le rythme de développement en Chine est bien supérieur au rythme français. Cela peut faire peur aux Français. Cela peut aussi créer un sentiment de... [décalage] que les anciennes générations d'étudiants chinois à l'étranger n'éprouvaient guère. À l'époque, pour ceux qui sont restés travailler en France après leur formation, quel que soit le métier qu'ils exerçaient, ils avaient une vie bien meilleure que ceux qui travaillaient en Chine. Car au début des années de la réforme et de l'ouverture (1978), jusqu'aux années 1990, le niveau de revenu en Chine était très bas, et les gens n'avaient pas autant de liberté qu'aujourd'hui. Aujourd'hui, nous faisons ce que nous voulons, n'est-ce pas ?

Maintenant, la Chine est devenue bien meilleure. Cela a créé un sentiment de... perplexité (miwang) chez les étudiants chinois à l'étranger. C'est-à-dire qu'on ne sait pas comment agir pour notre futur. Ceux qui ont commencé à travailler et qui veulent réussir éprouvent ce sentiment de décalage (chaju) ; ce sentiment de décalage serait encore plus grand pour ceux qui n'ont jamais travaillé. On a le sentiment qu'on a gâché notre vie, notre argent en France sans obtenir le moindre résultat. On a honte de soi en se comparant avec les autres ». (28 décembre 2008)

Le constat de retard crée donc un double décalage chez les migrants : d'abord, un décalage avec l’idéal de la réussite auquel on s'accroche. Nous pouvons parler de «l'éloignement des horizons d'attente », pour reprendre les termes de Paul Ricœur. Ensuite, un décalage avec autrui. Nous pouvons parler du «rétrécissement de l'espace d'expérience » ${ }^{40}$. Plus le migrant se trouve en retard par rapport à ses pairs en Chine, plus il éprouve un sentiment intense d'éloignement et d'étrangeté vis-à-vis de son pays d'origine. Il a du mal à « donner sens » à ses interactions avec ses compatriotes.

Dans ce contexte, nous comprenons l'ambivalence de nos enquêtés face au retour, après quelques années de travail en France. Beaucoup sont tentés par le retour dans leur pays d’origine, mais éprouvent en même temps une peur de ce retour. Lorsque la migration pour études ne garantit plus une position d'élite dans le pays d'origine, retourner en Chine et occuper une

39 Zheng avait travaillé pendant deux ans en Chine avant de venir étudier en France.

40 Ricœur, Paul,Temps et récit.Tome III, Le temps raconté, Paris : Seuil, 1985, 426 p. 
position inférieure à celles de leurs pairs non-migrants est synonyme d'échec, chose difficilement acceptable pour nos enquêtés et leurs proches ${ }^{41}$.

Effectivement, en suivant nos enquêtés dans l'évolution de leurs parcours, nous pouvons observer que certains migrants salariés sont retournés en Chine en développant différentes stratégies de reclassement social : la création de leurs propres entreprises en Chine, le retour avec un contrat d'expatriation d'une entreprise française, etc. D'autres migrants semblent s’installer durablement sur le sol français. L'accumulation des expériences de travail en France s’accompagne inévitablement d'un vieillissement biologique et social et par conséquent de nouvelles contraintes, notamment familiales, pour se mouvoir. Mais le migrant ne peut assumer le changement radical de son projet de vie sans changer sa structure de soi. Les expériences de décalages sont aussi occasion de retournement sur soi, de redéfinir le sens de sa migration en France, de prendre une distance critique avec les normes de la réussite sociale en Chine. Nous pouvons observer que la dimension de subjectivation ${ }^{42}$ commence à prendre le dessus chez les migrants qui ont acquis un degré minimum de la stabilité en France : ceux-ci sont de plus en plus préoccupés par la question de «style de vie », plutôt que par la question de « chances de vie $»^{43}$. En même temps, ils projettent leur idéal d'émancipation sur leur progéniture.

Pour notre paysagiste Zhou, lorsque sa chance objective de retourner en Chine s'amenuise, sa priorité est désormais de soutenir son épouse dans sa carrière professionnelle (elle deviendra maitre de conférences dans une université parisienne deux ans après notre premier entretien), mais aussi de créer un environnement favorable à l'épanouissement de l'enfant. Sa fille, qui aura grandi en France, sera " essentiellement une Française ». En tant que parent, sa vie sera bâtie en France, même si comme beaucoup de migrants, il pense à retourner en Chine à la retraite. Zhou disait :

« Avant, nous avions l'intention de gagner beaucoup d'argent. Effectivement, nous y avions pensé. Aujourd'hui, certains de mes camarades de classe sont déjà devenus les directeurs des instituts de design (en Chine). Ils ont de très hauts salaires, plus élevés que nos salaires d'ici (rire). Donc nous nous disons : gagner de l'argent, ce n'est pas essentiel. L'essentiel est d'avoir un environnement plus favorable à notre enfant. Premièrement, la compétition scolaire n'est pas aussi intense [qu'en Chine]. Deuxièmement, l'environnement naturel [y est plus favorable]. Il y a ces deux raisons... Et puis... pour la nourriture... Il n'y a pas le problème d'insécurité alimentaire en France » (5 mai 2014).

\section{Conclusion}

Dans cet article, nous avons souligné l'importance d'analyser les évaluations subjectives de la mobilité des diplômés chinois en France. En effet, c'est en comparaison avec leurs groupes de pairs non-migrants en Chine que nos enquêtés éprouvent paradoxalement un sentiment

\footnotetext{
41 Bolzman, Claudio; Gakuba, Théogène-Octave; Amalaman, Martin, "Honte et migration : une relation complexe à saisir”. Pensée plurielle, vol.44, $\mathrm{n}^{\circ}$ 1, pp.129-138; Pina-Guerassimoff, Carine, Circulation migratoire et mobilité internationale des migrants chinois. Migrations Société, vol.15, n 86, 2003, pp.9-22.

42 Martuccelli, Danilo,Grammaires del'individu,Paris : Editions Gallimard, 2002, 712 p.

${ }^{43}$ Giddens, Anthony,Modernityandself-identity: self and society in the late modern age. Stanford, CA: University Press, 1991, 256 p.
} 
intense d'immobilité, même s'ils développent, d’un point de vue socioprofessionnel, des trajectoires ascendantes à l'échelle intragénérationnelle.

Au cœur de ce sentiment d'immobilité se retrouve l'expérience temporelle de nos enquêtés dans le processus biographique et dans le contexte des changements sociaux rapides en Chine. Tout d'abord, nos enquêtés se considèrent en retard dans leur entrée dans la vie adulte (notamment le mariage) au regard des normes sociales relatives à l'âge en Chine. Deuxièmement, nos enquêtés se voient devancer par leurs pairs en Chine en ce qui concerne leur progression sur une échelle sociale (accès à la promotion professionnelle, accession à la propriété immobilière, etc.), en raison des rythmes différents des changements sociaux dans le pays de départ et dans le pays d'arrivée qui joue en faveur des ceux qui sont « restés » en Chine.

Les effets conjoints de ces deux dynamiques créent chez les migrants étudiants chinois un double décalage, par rapport à leur idéal de réussite et par rapport à leurs pairs non-migrants. Ce double décalage va engendrer chez les migrants un sentiment d'échec, une honte de soi et une peur de retour. Cette «honte », combinée au processus biographique et au processus de subjectivation, conduisent certains enquêtés à réviser leur projet de migration en faveur d'une installation sur le sol français. Finalement, pour les jeunes chinois que nous avons étudiés, le potentiel transformateur de la mobilité pour études réside moins dans son rôle «d'ascenseur social » que dans sa force de changer le projet de vie des migrants et corollairement leur définition de soi. 